\title{
The MAX QUASI-INDEPENDENT SET problem
}

\author{
N. Bourgeois ${ }^{1}$, A. Giannakos ${ }^{1}$, G. Lucarelli² , I. Milis², V. Th. Paschos' ${ }^{1}$, O. Pottié ${ }^{1}$ \\ ${ }^{1}$ LAMSADE, CNRS and Université Paris-Dauphine \\ \{bourgeois, giannako, paschos, pottie\}@lamsade.dauphine.fr \\ ${ }^{2}$ Department of Informatics, Athens University of Economics and Business
}

\{gluc,milis\}@aueb.gr

September 3, 2009

\begin{abstract}
In this paper, we deal with the problem of finding quasi-independent sets in graphs. This problem is formally defined in three versions, which are shown to be polynomially equivalent. The one that looks most general, namely, $f$-QIS, consists of, given a graph and a non-decreasing function $f$, finding a maximum size subset $Q$ of the vertices of the graph, such that the number of vertices in the induced subgraph is less than or equal to $f(|Q|)$. For this problem, we show an exact solution method that runs within time $O^{*}\left(2^{\frac{d-27 / 23}{d+1} n}\right)$ on graphs of average degree bounded by $d$. For the most specifically defined $\gamma-\mathrm{QIS}$ and $k-\mathrm{QIS}$ problems, several results on approximation are shown, and greedy algorithms are proposed, analyzed and tested; we also prove some polynomial cases.
\end{abstract}

\section{Introduction and preliminaries}

The problem of finding in a graph a maximum-size subgraph whose density differs (being smaller or larger) from that of the whole graph, arises often in various application contexts. For example, inputs may represent graphs, wherein dense (with respect to the input) subgraphs are sought, as it is the case for call details database mining [1], or protein-protein interaction networks analysis [8]. In other cases, inputs may represent graphs from which one wants to extract maximum size, sparser than the input graphs, as for example in visualisation tools for stock market interaction graphs [3].

In this paper we tackle problem of finding in a graph a maximum size subgraph whose sparsity is less than or equal to a value specified with the input. In the case that appears as most general, the sparsity of a graph is measured by means of a function bounding the number of edges in the sought subgraph and depending on its size; we also study some special forms of this function, namely, when it has the form of the ratio of the number of edges of the solution to the number of edges in a complete graph of equal size, and also when it is a numeric parameter of the input.

We note by $G$ a simple finite graph without loops, by $V$ and $E(G)$ its vertex set and its edge set, respectively, and by $n$ and $m$ their respective sizes. Let $A, B$ be two subsets of $V$. The induced subgraph by $A$ in $G$ is denoted by $G[A]$ and its edge set by $E[A]$, respectively. The edge set with one extremity in $A$ and the other in $B \backslash A$ will be denoted as $E[A, B]$. Clearly, if $A$ and $B$ are disjoint then $E[A, B]=E[B, A]$ and $E[A, A]=\emptyset$. The degree of $A$ towards $B$ is equal to $|E[A, B]|$ and is denoted by $\delta_{B}(A)$; when $A$ is reduced to a singleton $\{v\}$, we note its degree in $B$ by $\delta_{B}(v)$, or simply by $\delta(v)$ whenever $B=V$. The maximum vertex-degree in a graph $G$ is denoted by $\Delta_{G}$ or simply by $\Delta$ if there is no risk of confusion. We also set $d_{B}(A)=1 /|A| \sum_{v \in A} \delta_{B}(v)$, and $d=1 / n \sum_{v \in V} \delta(v)$.

In this paper we tackle the following variants of the quasi-independent set problem.

Maximum $f$-Quasi-INDEPENDENT Set ( $f$-QIS, the general quasi-independent set problem):

Given a graph $G$ and a polynomially computable non-decreasing real function $f: \mathbb{N} \rightarrow \mathbb{R}$, find the largest possible $Q \subseteq V$ such that $\mid E[Q]) \mid \leq f(|Q|)$.

In the above definition, $f$ is used as a sparsity specification for the induced subgraph of the sought solution.

In this paper, we study in particular two variants of $f$-QIS, denoted by $\gamma$-QIS and $k$-QIS, respectively, formally defined in what follows. 
In the first one sparsity specification is given in the special form of the ratio of the number of edges in the subgraph induced by the quasi-independent set over the number of edges induced by a complete graph of the same size:

Maximum $\gamma$-Quasi-IndePendent Set $(\gamma$-QIS):

Given a graph $G$ and a real $\gamma, 0 \leq \gamma \leq 1$, find the largest possible $Q \subseteq V$ such that $\mid E[Q]) \mid \leq \gamma\left(\frac{|Q|}{2}\right)$.

It is easy to see that $\gamma$-QIS is not hereditary (a problem is said hereditary if its solutions satisfy some non-trivial hereditary property $\left.{ }^{1}\right)$. Indeed, given a fealible solution for $\gamma$-QIS, it may be that the induced subgraph $G\left[Q^{\prime}\right]$ of a subset $Q^{\prime}$ of $Q$ violates the sparsity condition $E\left(Q^{\prime}\right) \leqslant \gamma\left|Q^{\prime}\right|\left(\left|Q^{\prime}\right|-1\right) / 2$.

The other restricted variant of the problem considered in the paper is the one where we simply seek for a maximum vertex subset of the graph with no more than a constant number of edges having both extremities in it:

Maximum $k$-QuAsi-IndePEndent Set ( $k$-QIS):

Given a graph $G$ and a positive integer $k$, find the largest possible $Q \subseteq V$ such that $\mid E[Q]) \mid \leq k$.

Clearly, $k$-QIS is hereditary. In fact, it is easy to see that $k$-QIS belongs to the family of node-deletion problems, first defined in [11] and further studied by Yannakakis ([15]). Formally, a node-deletion problem consists of finding, given a graph $G$ and a non-trivial hereditary property $P$, the minimum number of vertices of $G$ that one has to delete from $G$, in order to have $P$ satisfied in the remaining graph. In [15], the author proves that the decision version of such problems is NP-complete even for planar graphs.

For $f \equiv 0$ (resp., $\gamma=0$ and $k=0$ ), $Q$ is simply a maximum independent set in $G$, while for $f: f(|Q|) \geq m$ (resp., for $\gamma \geq 2 m / n(n-1)$ and $k \geq m$ ), $Q=V$ is a trivial solution; being a direct generalization of the MaXimum InDEPENDENT SeT, the $f-, \gamma-$ and $k$ - QIS problems are obviously inapproximable within better than $O\left(n^{1-\epsilon}\right)$, unless $\mathbf{P}=\mathbf{N P}$ [17].

J. Abello, M.G.C. Resende, and S. Sudarsky in [1] address essentially the same problem, formulated as the research, given a graph and $0 \leq \gamma \leq 1$, of a maximum subgraph of sparsity (defined as the ratio of the number of its edges over the number of edges of the complete graph of the same size) greater than or equal to $\gamma$; any solution to this problem can be obtained as the complementary of a quasi-independent set of sparsity $\leq 1-\gamma$ in the complementary of the input graph. The authors present an algorithm equivalent to the greedy algotihm for $\gamma$-QIS, analyzed later in this paper; however, they focus in implementation issues on very large instances and they don't attempt to analyze its performance.

A. Jagota, G. Narasimhan and Ľ. Šoltés give in [10] an alternative generalization of independent sets, namely $k$-insulated sets, which are subsets of the graph vertex set such that any vertex in a $k$-insulated set is adjacent to at most $k$ vertices in it, while any external vertex is adjacent to at least $k+1$ vertices of the $k$-insulated set.

To our knowledge, the $k$-QIS problem has been specifically formulated for the first time by D.S. Hochbaum and O. Goldschmidt in [9]. The authors call it the k-edge-in subgraph problem. Nevertheless, in this paper the problem is not addressed, but simply mentioned as related to other subgraph problems on which it focuses.

A kind of dual of the maximum quasi-independent set problem would be to search, given a graph and a positive integer $k$, for the sparsest - or densest - possible (maximal) subgraph with $k$ vertices. This kind of problems has extensively been studied during the last years under the names of " $k$-sparsest" or " $k$-densest subgraph" problem (see, for example, [?]). Similar problems have been studied in previous works has by A.V. Goldberg [5] and by J.C. Picard and M. Queyranne [13]. However, these papers define sparsity as the ratio of a positive power of the size of a graph over the number of its induced edges.

The remainder of the paper is organized as follows. Section 2 gives several bounds to the optimal solutions for $\gamma$-QIS. Section 3 tackles specific polynomial cases for the three variants of MAX QUASI-INDEPENDENT SET and proves its NP-hardness in bipartite graphs. In Section 4 an exact solution method with non-trivial worst-case running time for the general $f$-QIS problem is presented and analyzed. As we discuss here this method applies also to other combinatorial optimization problems. Finally, in Section 5 approximation results are proved for both $\gamma$-QIS (Subsection 5.1) and $k$-QIS (Subsection 5.2).

In what follows, when we indifferently refer to either one of the quasi-independent set versions defined above, we use the term MAX QUASI-INDEPENDENT SET instead. Also, when no confusion arises, we sometimes use $f-$, or $\gamma-$, or $k$-QIS in a twofold way: either to speak about the problem itself, or to speak about a feasible solution of the corresponding problem.

\footnotetext{
${ }^{1}$ A graph $G$ is said to satisfy a hereditary property $\pi$ if every subgraph of $G$ satisfies $\pi$ whenever $G$ satisfies $\pi$. Furthermore, $\pi$ is non-trivial if it is satisfied for infinitely many graphs and it is false for infinitely many graphs; for instance, properties "independent set", "clique", "planar graph", "k-colorable graph", etc., are non-trivial hereditary properties.
} 


\section{Solution properties and bounds}

As it is already mentioned, in general, $\gamma$-QIS is not a hereditary problem; just consider an instance where the graph is an edge plus some isolated vertices, and $\gamma$ is the smallest possible for having the whole graph as a trivial solution. Obviously, the sparsity condition will be violated for any strict part of the solution containing the edge. However, $\gamma$-QIS is still a weakly hereditary problem, in the sense given by the following lemma that will be used later.

Lemma 1 Let $Q$ be a $\gamma$-QIS in $G$, of size $q$. Then, for any $k \leq q$, there exists in $G$ some $\gamma-Q I S R(k) \subseteq Q$, of size $k$.

Proof. Let $p<q$ be the maximum value such that the property is false. Consider some $R(p+1)$; for any $v \in R(p+1)$ it is:

$$
\frac{\gamma p(p-1)}{2}<|E[R(p+1) \backslash v]|
$$

Summing up these inequalities, we get:

$$
\frac{\gamma p(p-1)(p+1)}{2}<\sum_{v \in R(p+1)}|E[R(p+1) \backslash v]|=(p+1)|E[R(p+1)]|-\sum_{v \in R(p+1)} \delta_{R(p+1)}(v) \leq \frac{\gamma(p-1) p(p+1)}{2},
$$

which is a contradiction.

Next lemma give some bounds for the solutions of the $\gamma$-QIS.

Lemma 2 Let $Q$ be any non-trivial $\gamma$ - quasi-independent set $\left(0<\gamma<m /\left(\begin{array}{c}n \\ 2\end{array}\right)\right)$, with size $q$, not contained in any $\gamma-$ quasi-independent set of size $q+1$. Let $\vartheta(Q)=\min _{v \in V \backslash Q}\left\{\delta_{Q}(v)\right\}$, let $Q^{*} \neq Q$ be an optimal solution for $\gamma-Q I S$, $q^{*}$ be its size and, for any vertex-subset $P$, let $d(P)$ be the average degree of the subgraph induced by $P$ (recall that we note $d(V)$ by $d$ ). Finally, let $\alpha_{\min }$ be the size of a smallest maximal independent set (minimum independent dominating set) in G. Then:

1. $q^{*} \leq \alpha_{\min } \Delta$

2. $\frac{q^{*}}{q} \leq \frac{\Delta}{9(Q)}$

3. $q \geq n-\frac{\Delta}{\gamma}$

4. $q^{*} \leq \frac{\Delta}{\gamma}$.

5. $q^{*} \leq \sqrt{\frac{d n}{\gamma}}$

6. $q^{*} \leq \frac{d\left(Q^{*}\right)+2}{\gamma}-1$.

Proof. Let $S$ be any maximal independent set in $G$, denote by $Q^{*}$ a quasi-independent set of maximum size and set $S^{\prime}=Q^{*} \cap S$. By the maximality of $S, q *-\left|S^{\prime}\right| \leq \delta_{S}\left(Q^{*}\right) \leq\left(|S|-\left|S^{\prime}\right|\right) \Delta$; hence $q^{*} \leq|S| \Delta \leq \alpha_{\min } \Delta$ and item 1 is proved.

We now prove item 2. By the definition of $Q$, it is $\vartheta(Q)>0$ and

$$
\vartheta(Q)(n-q) \leq \delta_{V}(Q)=\sum_{v \in Q} \delta(v)-2 E[Q] \leq \Delta q
$$

By a similar argument, it is $\vartheta(Q)\left(q^{*}-\left|Q^{*} \cap Q\right|\right) \leq \delta_{Q \backslash Q^{*} \cap Q}\left(Q^{*} \backslash Q^{*} \cap Q\right) \leq \Delta\left(q-\left|Q^{*} \cap Q\right|\right)$, hence

$$
\frac{q^{*}}{q} \leq \frac{q^{*}-\left|Q^{*} \cap Q\right|}{q-\left|Q^{*} \cap Q\right|} \leq \frac{\Delta}{\vartheta(Q)}
$$

Proof of item 3. By the definition of $Q$, we have also that

$$
\gamma \frac{q(q+1)}{2}<E[Q]+\vartheta(Q) \leq \gamma \frac{q(q-1)}{2}+\vartheta(Q) \Rightarrow \vartheta(Q)>\gamma q
$$


Combining (1) and (3) we get $\gamma q(n-q) \leq \Delta q \Rightarrow q \geq n-\frac{\Delta}{\gamma}$, that proves item 3, while combining (2) and (3) we get immediately $q^{*} \leq \frac{\Delta}{\gamma}$ proving so item 4 .

Assuming that $Q^{*}$ is non-trivial, we get $\frac{\gamma q(q+1)}{2}<m=\frac{d n}{2} \Rightarrow \frac{\gamma q^{2}}{2}<\frac{d n}{2} \Leftrightarrow q^{*} \leq \sqrt{\frac{d n}{\gamma}}$, that proves item 5 , and also that $\frac{\gamma q(q+1)}{2}<E[Q \cup v] \leq E[Q]+q=\frac{d(Q) q}{2}+q \Leftrightarrow \gamma(q+1)<d(Q)+2 \Rightarrow q^{*}<\frac{d\left(Q^{*}\right)+2}{\gamma}-1$ that proves item 6 and completes the proof of the lemma.

\section{Complexity results for MAX QUASI-INDEPENDENT SET in various graph- classes}

\subsection{Relations between $f-, \gamma-$ and $k$-QIS}

We show in this section that all three variants of MAX QUASI-INDEPENDENT SET dealt in this paper are closely interrelated. This is proved in the following proposition.

Proposition $1 f$-QIS, $\gamma$-QIS and k-QIS are polynomially equivalent with respect to their exact solution.

Proof. (i) We first show that $\gamma$-QIS $\leq f$-QIS. This is trivial since the condition for sparsity formed as expression of $\gamma$ is, as noted before, just one particular case of a sparsity specification defined by $f(q)=$ $\gamma q(q-1) / 2$.

(ii) We now show that $f$-QIS $\leq k$-QIS: indeed, consider some instance $I=(G, f)$ of the $f$-QIS problem, and let $Q^{*}(I)$ be any of its optimal solutions; note its size by $q^{*}(I)$. Let $\left|E\left[Q^{*}(I)\right]\right|=k^{\prime} \leq f\left(q^{*}(I)\right)$ be the number of edges in this optimal; clearly, $Q^{*}(I)$ should be also an optimal solution for the instance $\left(G, k^{\prime}\right)$ of the $k-Q I S$ problem, ie some instance $(G, i)$ with $0 \leq i \leq m$. Thus, $q^{*}(I)$ has to be the size of one of the optimal solutions $Q^{*}(G, i)$ of the family of instances $\{(G, i): 0 \leq i \leq m\}$, more specifically of such an optimal that it satisfies $Q^{*}(G, i) \in \operatorname{argmax}_{i}\left\{q^{*}(G, i):\left|E\left[Q^{*}(G, i)\right]\right| \leq f\left(q^{*}(G, i)\right)\right\}$. Since there are at most $m$ of $k$-QIS instances to solve, the claim follows.

(iii) Now it remains to show that $k-\mathrm{QIS} \leq \gamma$-QIS. In a similar manner as before, consider some instance $I=(G, k)$ of the $k$-QIS, and let $Q^{*}(I)$ be any of its optimal solutions; note its size by $q^{*}(I)$. Let $\left|E\left[Q^{*}(I)\right]\right|=k^{\prime} \leq k$ be the number of edges in this optimal; clearly, $Q^{*}(I)$ must also be an optimal solution for some instance $\left(G, \gamma\left(q^{*}(I), k^{\prime}\right)\right)$ with $\gamma\left(q^{*}(I), k^{\prime}\right)=\frac{2 k^{\prime}}{q^{*}(I)\left(q^{*}(I)-1\right)}$ of the $\gamma$-QIS problem; notice that $0 \leq k^{\prime} \leq k \leq m$ and w.l.o.g. $2 \leq q^{*}(I) \leq n$. Thus, $q^{*}(I)$ has to be the size of one of the optimal solutions $Q^{*}(G, \gamma(i, j))$ of the family of instances of $\gamma$-QIS, $\left\{(G, \gamma(i, j)): \gamma(i, j)=\frac{2 i}{j(j-1)}, 0 \leq i \leq k, 2 \leq j \leq n\right\}$, more specifically of such an optimal that it satisfies $Q^{*}(G, \gamma(i, j)) \in \operatorname{argmax}_{0 \leq i \leq m, 2 \leq j \leq n}\left\{q^{*}(G, \gamma(i, j)):\left|E\left[Q^{*}(G, \gamma(i, j))\right]\right| \leq k\right\}$. Since there are at most $n m$ instances of the $\gamma$-QIS to solve, the claim follows.

Combining all the above derives the proposition's claim.

\subsection{Bipartite graphs}

We now tackle $k$-QIS in bipartite graphs. Our goal is to prove the following proposition.

Proposition 2 -QIS problem is NP-hard on bipartite graphs.

Proof. Our reduction goes from the $r$-SPARSEST SUBGRAPH problem, which consists of seeking, given a graph $G(V, E)$ and an integer $1<r<n$, a subset of $H \subset V$ of size $r$ such that $G[H]$ has a minimum number of edges, among all induced subgraphs of size $r$. In the decision version of $r$-SPARSEST SUBGRAPH, we are given $G(V, E)$ and two positive integers $r$ and $l$ and we ask if there is $H \subset V$ of size $r$ inducing a subgraph with at most $l$ edges; this problem is NP-complete for bipartite graphs [16]. Consider the decision version of $k-Q I S:$ given $G$ and two positive integers $t$ and $k$, is there $Q \subseteq V$ such that $|Q| \geq t$ and $G[Q]$ has at most $k$ edges?

It is straightforward to see that given an instance $I=(G, r, l)$ of (the decision version of) $r$-SPARSEST SUBGRAPH, the instance $I^{\prime}=(G, t=r, l=k)$ of $k$-QIS is a "yes"-instance if $I$ is a "yes"-instance too. On the other hand, if $I^{\prime}$ has a solution, say $Q^{*}$ with $\left|Q^{*}\right|>r$, one can easily get a solution for $I$, just by eliminating any $\left|Q^{*}\right|-r$ vertices from $Q^{*}$ (recall that $k-Q I S$ is hereditary).

Combining Propositions 1 and 2, the following result holds that shows that MAX QUASI-INDEPENDENT SET is quite different from the classical MAX INDEPENDENT SET problem (that is polynomial in bipartite graphs). 
Corollary $1 \gamma$-and $f$-QIS are NP-hard even in bipartite graphs.

There are several hereditary graph classes the definitions of which imply direct conditions on their sparsity, independently of the measure used; take for instance complete graphs or trees. Such proprieties, together with heredity, can be exploited in order to polynomially solve the $\gamma$-QIS (in fact, by Proposition 1, any of the three variants of MAX QUASI-INDEPENDENT SET). In the sequel, we present two polynomial algorithms for $\gamma$-QIS, that work in forests and split graphs, respectively.

\subsection{Forests}

Let $F=(V, E)$ be a forest. It is $|E|=n-c$, where $c$ is the number of the connected components (for a single tree $T(V, E)$, it is $|E|=n-1)$. This means that, we can suppose w.l.o.g. that $\gamma \leq \frac{2(n-c)}{n(n-1)}$, since otherwise the whole graph will be the trivial optimal solution.

Hence, the following algorithm can be proposed for $\gamma$-QIS in forests:

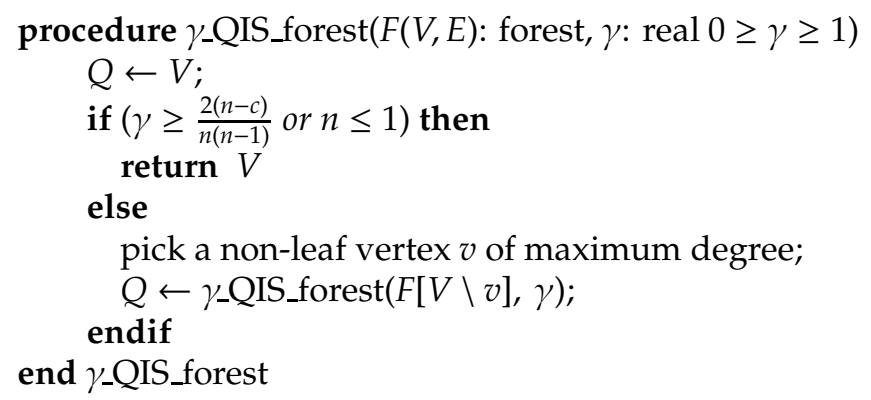

Theorem 1 Algorithm $\gamma_{-} Q I S_{-}$forest $(F(V, E)$ finds an optimal $\gamma-Q I S$ in polynomial time.

Proof. Let $Q^{*}$ be an optimal solution and $q^{*}$ its size. Obviously, $F\left[Q^{*}\right]$ must be a forest with at most $n-\frac{\gamma q(q-1)}{2}$ connected components. But $\gamma_{-}$QIS_forest $(F(V, E)$ just tests if $F$ has a trivial solution and if not, removes a vertex such that the number of connected components in the remaining graph be maximized.

Combining Proposition 1 and Theorem 1, the following result is immediately derived.

Corollary 2 All $f-, \gamma$ - and $k-Q I S$ are polynomial in forests.

\subsection{Split graphs}

Let $S=(I, C, E)$ be a split graph, where $I$ is an independent set and $C$ is a clique.

Lemma 3 There is an optimal $\gamma$-QIS in a split graph $S=(I, C, E)$ such that it contains the independent set $I$.

Proof. Let $I^{\prime} \cup C^{\prime}, I^{\prime} \subset I, C^{\prime} \subseteq C$, be the set of vertices selected by an optimal solution. If we remove a vertex $c \in C^{\prime}$ from this optimal we remove also $C^{\prime}-1$ edges. If we next add a vertex $i \in I \backslash I^{\prime}$ we add at most $C^{\prime}-1$ edges.

Thus, the following theorem holds:

Theorem 2 The optimal solution to the quasi independent set problem in a split graph $S=(I, C, E)$ can be found in polynomial time.

Proof. It suffices to use $\gamma$-QIS with $S$ initialized to I; by Lemma 3, the proof is straightforward.

Corollary 3 All $f-, \gamma$ - and $k-Q I S$ are polynomial in split graphs.

\section{EXact solution of MAX QUASI-INDEPENDENT SET}

We give in this section an exact algorithm for $\gamma$-QIS with non-trivial worst-case running time. Let us note that to our knowledge, no algorithm that optimally solves MAX QUASI-INDEPENDENT SET with running time better than $O^{*}\left(2^{n}\right)$ is known. Also, as we will see in the sequel, the scope of the results of this section is even larger than the MAX QUASI-INDEPENDENT SET-case. Indeed, the method described in what follows concerns a broad class of optimization problems, those that "match vertex branching", defined in Definition 1, below. 


\subsection{Problems that match vertex branching}

The intuition behind the exact solution method for MAX QUASI-INDEPENDENT SET, lies in the possibility of organizing the solution space of the problem in a tree-like manner. So we need first to formally characterize the class of optimization graph-problems for which such an organization is possible. This is done in Definition 1.

Definition 1 We say that a graph problem $\Pi$ matches vertex branching, if for any graph instance $G(V, E)$, for any $v \in V$, there exist some sets of parameters $\mathcal{S}_{i}$, some subsets $v \in H_{i} \subset V$ and two functions $f_{1}, f_{2}$ bounded above by some polynomial of $n$, such that:

$$
o p t_{\Pi}\left(G, \mathcal{S}_{3}\right) \leq \max \left\{f_{1}\left(o p t_{\Pi}\left(G\left[V \backslash H_{1}\right], \mathcal{S}_{1}\right)\right), f_{2}\left(o p t_{\Pi}\left(G\left[V \backslash H_{2}\right], \mathcal{S}_{2}\right)\right)\right\}
$$

where opt ${ }_{\Pi}(G, \mathcal{S})$ denotes the optimal solution of $\Pi$ for $G$ with parameter set $\mathcal{S}$.

Notice that, with appropriate choice for $f_{1}, f_{2}$, it is possible to replace max by min, or to make $k>2$ branchings, or a single reduction (this last case corresponds to polynomial problems).

Most of the problems whose aim is to find a specific subset in a given graph may be generalized as a problem that matches vertex branching. For exemple, for the MAXimum Weighted Independent Set: Given a graph $G(V, E)$ and a weight function $w: V \rightarrow \mathbb{R}$, we search for an independent set $S$ maximizing $\sum_{v \in S} w(v)$, it is:

$$
\operatorname{opt}(G, w) \leq \max \{\operatorname{opt}(G[V \backslash v], w), \operatorname{opt}(G[V \backslash N[v]], w)+w(v)\}
$$

Obviously, this remains true for the non-weighted version, ie whenever $w \equiv 1$.

Also the $f$-QIS can be reformulated as a problem that matches vertex branching, in the following manner: Given a graph $G(V, E)$, two constants $w_{0}, q_{0}$ and a weight function $w: V \rightarrow \mathbb{R}$, we search for a maximal size vertex subset $Q \subseteq V$ whose induced graph $G[Q](Q, R)$ verifies $|R|+w_{0}+\sum_{v \in Q} w(v) \leq f\left(|Q|+q_{0}\right)$. Let $w_{+} \equiv w+1$ on $N(v)=\{u:\{u, v\} \in E\}$ and $w_{+} \equiv w$ elsewhere. Then:

$$
\operatorname{opt}\left(G, w_{0}, q_{0}, w\right) \leq \max \left\{\operatorname{opt}\left(G[V \backslash v], w_{0}, q_{0}, w\right), \operatorname{opt}\left(G[V \backslash v], w_{0}+w(v), q_{0}+1, w_{+}\right)\right\}
$$

and the formulation is completed by setting initially $q_{0}=w_{0}=0, w \equiv 0$.

Notice that, as it can be shown by straightforward recurrence, any problem that matches vertex branching can be solved within time $O\left(2^{n} \times\right.$ poly $\left.(n, k)\right)$, where $\left|\mathcal{S}_{i}\right|<k$. Obviously, this is useless for problems where a specific subgraph is sought, since they can be solved in $O^{*}\left(2^{n}\right)$.

However, an exact algorithm for $f$-QIS based upon vertex branching would be interesting if its running time $T(n)$ could be shown to be in $2^{\phi(\Delta) n}$ with $\phi$ some increasing function bounded above by 1 for any $\Delta$. Intuitively, a possibility for such an improvement lies in finding an efficient vertex branching rule for fastly diminishing the remaing graph's degree, and showing fast (polynomial) algorithms for computing a maximum $f$-QIS problem in bounded degree graphs.

\subsection{Bottom-up Algorithms}

\subsubsection{General scheme}

We give below a general scheme, using vertex branching for finding a maximum $f$-QIS in a graph G. Recall that by Proposition 4 such a method can be used for computing an optimal solution for any of the three variants of the MAX QUASI-INDEPENDENT SET problem, with a polynomial overhead.This scheme can be written as follows:

procedure exactrec $\left(G(V, E)\right.$ : graph, $f$ : integer function, $q_{0}, w_{0}$ : integer, $w$ : vertex weight function)

in, not_in: integer;

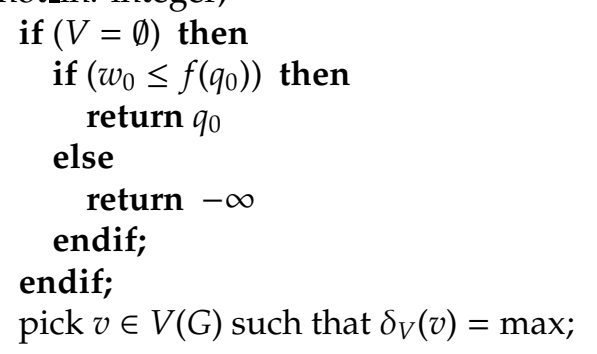




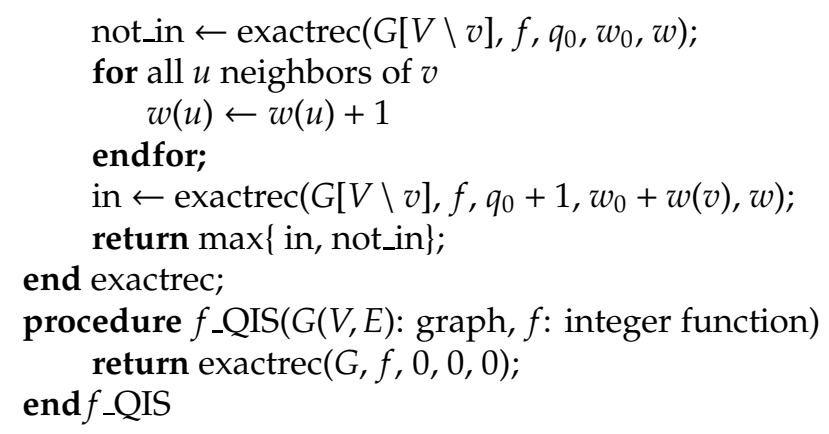

As noted at the end of the previous subsection, the running time for an exact method induced by the above scheme can be accelerated if the $f$-QIS problem is shown polynomial on bounded small degree graphs. This is established by the following series of propositions:

Lemma 4 Assume that some problem that matches vertex branching can be computed on graphs whose average degree is at most $d-1$, within time $O^{*}\left(2^{\alpha_{d} n}\right)$ for a given $\alpha_{d}$. Then, it can be computed on graphs whose average degree is at least $d-1$ within time $O^{*}\left(2^{\alpha_{d} n+\beta_{d}(m-(d-1) n / 2)}\right)$, where

$$
\beta_{d}=\frac{2\left(1-\alpha_{d}\right)}{d+1}
$$

Proof. Let $T(m, n)$ be the (worst case) complexity of solution for instances of size $n$ and of $m$ edges. We proceed by induction on $m, n$. Trivially, the hypothesis of the statement holds for $m_{0}=(d-1) n / 2$. Suppose that it is true for any pair $n^{\prime}<n, m^{\prime}<m$. Since the graph has average degree greater than $d-1$, there exists some vertex of degree $d$ or more. When branching on it, we get:

$$
\begin{aligned}
T(m, n) & \leq 2 T(m-d, n-1) \\
& \leq 2^{1+\alpha_{d}(n-1)+\beta_{d}(m-(d-1) n / 2)-(d+1) / 2} \\
& \leq 2^{1-\alpha_{d}-(d+1) / 2 \times 2\left(1-\alpha_{d}\right) /(d+1)} \times 2^{\alpha_{d} n+\beta_{d}(m-(d-1) n / 2)} \\
& \leq 2^{\alpha_{d} n+\beta_{d}(m-(d-1) n / 2)}
\end{aligned}
$$

that completes the proof.

As a straightforward consequence of the above lemma, the following proposition holds:

Proposition 3 Assume that some problem that matches vertex branching can be computed on graphs whose average degree is at most $d-1$ within time $O^{*}\left(2^{\alpha_{d} n}\right)$ for a given $\alpha_{d}$. Then, it may be computed on graphs whose average degree is at most $d$ within time $O^{*}\left(2^{\alpha_{d+1} n}\right)$, where

$$
\alpha_{d+1}=\frac{d \alpha_{d}+1}{d+1}
$$

As a direct consequence of Proposition 3, comes the following theorem:

Theorem 3 Any problem that is polynomial on totally disconnected graphs and matches vertex branching can be solved on graphs of average degree at most $d$ with running time $O^{*}\left(2^{\text {dn/(d+1) }}\right)$.

It is also possible to give a very simple direct proof based on a well-known corollary of Turan's Theorem, namely that for a graph of average degree $d=2 \mathrm{~m} / n$ and of maximum independent set size $\alpha^{*}$ :

$$
\alpha^{*} \geq \frac{n^{2}}{2 m+n}=\frac{n}{d+1}
$$

Proof. One can run some exact algorithm for max InDEPEndent SET (for instance, the one in [4], that has complexity is bounded by $O^{*}\left(2^{0.288 n}\right)$ ). Let $S^{*}$ the computed solution. Branch on any vertex belonging to its complementary, $V \backslash S^{*}$; the remaining graph is totally disconnected, so it can be solved in polynomial time.

Thus, the total running time will be in $O^{*}\left(2^{0.288 n}+2^{n-\alpha(G)}\right) \subset O^{*}\left(2^{\frac{d}{d+1} n}\right)$ 
Notice that this bound is tight for a greedy choice of branching, ie if the algorithm always branches on a vertex of largest degree among the remaining ones, and for branching on an independent set of maximal size.

To illustrate the tightness, consider for any $\delta \leq d$ the graph $G_{\delta}$ that is composed of $n /(d+1)$ cliques of size $\delta+1 . T\left(G_{1}\right)=2^{n /(d+1)}$. The algorithm removes one vertex in each connected component, so we have $T\left(G_{\delta}\right)=2^{n /(d+1)} T\left(G_{\delta-1}\right)$ and finally $T\left(G_{d}\right)=2^{d n /(d+1)}$ On the other hand, $\alpha\left(G_{d}\right)=n /(d+1)$ (one vertex per clique).

Unfortunately, there is little hope for generalizing this corollary, since no problem is polynomial on graphs of average degree bounded above by some $d>0$, unless it belongs to $\mathbf{P}$ (just add some independent set to decrease $d$ ). Furthermore, restricting the instance set to graphs without isolated vertices, or even to connected graphs, does not help much, since the greedy branching may disconnect the graph as well. On the other hand, some improvement results can be obtained for graphs of bounded maximum degree.

\subsubsection{Using polynomial solution on graphs of maximum degree 2}

Many problems that match vertex branching are in fact well-known to be polynomial on graphs of maximum degree 2, for instance MAX INDEPENDENT SET (or equivalently MAX CLIQUE and MIN VERTEX COVER). For some difficult problems like MAX QUASI-INDEPENDENT SET that remains true, but that is not straightforward; we give a proof in subsection 4.3.

Proposition 4 Any problem that is polynomial on graphs of maximal degree 2 and matches vertex branching can be solved on graphs of average degree $d$ with running time $O^{*}\left(2^{d n / 6}\right)$. This bound is tight for $d=3$ and a greedy choice of the branching.

Proof. If there is no vertex of degree 3 or more, the problem can be solved in polynomial time. Otherwise, when branching on some vertex of maximal degree, we remove at least 3 edges from the graph, that means $T(m) \leq 2 T(m-3)$, that leads to

$$
T(m)=O^{*}\left(2^{m / 3}\right)=O^{*}\left(2^{d n / 6}\right)
$$

To show the tightness, consider a graph composed of $n / 6$ copies of $K_{3,3}$. In any connected component, the algorithm greedily branches on three vertices, so $T(n)=2^{n / 2}$.

Proposition 5 Any problem that is polynomial on graphs of maximal degree 2 and matches vertex branching can be solved on graphs of average degree that rounds up to $d \leq 2$ with running time $O^{*}\left(2^{\frac{d-1}{d+1} n}\right)$. If the average degree is $d$, this bound is tight for a greedy choice of the branching.

Proof. In a notation like the one introduced in Proposition 3, this means that we claim $\alpha_{d+1}=(d-1) /(d+1)$. As a consequence of Proposition 4 with $d \in\{2,3\}$, we see that any problem which is polynomial on graphs of maximal degree 2 and matches vertex branching can be solved on graphs of average degree 2 (resp. 3) within time $O^{*}\left(2^{n / 2}\right)\left(\right.$ resp. $\left.O^{*}\left(2^{n / 3}\right)\right)$. Thus, our hypothesis is verified for $d=2$ and 3 .

Now assume that the statement holds for $d-1$. Then, according to Proposition 3,

$$
\alpha_{d+1}=\frac{d \frac{d-2}{d}+1}{d+1}=\frac{d-1}{d+1}
$$

and the result yields by recurrence.

In order to prove tightness, we form the graph $G_{\delta}^{\prime}$ in the following way:

- $G_{3}^{\prime}$ is composed of $\frac{2 n}{3(d+1)}$ copies of $K_{3,3}$. $T\left(G_{3}^{\prime}\right)=2^{2 n(d+1)}$

- Partition each pair of copies of $K_{3,3}$ into three subsets of size 4 , namely $A_{1}, A_{2}, A_{3}$. For any $i$ add a vertex $a_{i, 4}$ adjacent to all the vertices in $A_{i}$. That is $G_{4}^{\prime}$.

- For any $\delta \leq d-1$, form $G_{\delta+1}^{\prime}$ by adding a vertex $a_{i, d+1}$ adjacent to all the vertices in $A_{i} \cup\left\{a_{i, 4}, \ldots, a_{i, d}\right\}$

The algorithm removes three vertices from each connected component, so we have $T\left(G_{\delta}^{\prime}\right)=2^{n /(d+1)} T\left(G_{\delta-1}^{\prime}\right)$ and finally $T\left(G_{d}^{\prime}\right)=2^{(d-1) n /(d+1)}$. 


\subsubsection{Performance of bottom-up for problems that match vertex branching, on graphs of bounded maximum degree}

First we deal with the case of graphs of maximum degree 3, followed by the general case of graphs with bounded maximum degree.

Proposition 6 Any problem that matches vertex branching and is polynomial on graphs of maximum degree 2 can be solved on graphs of maximum degree 3 within time $O^{*}\left(2^{3 n / 8}\right)$.

Proof. Notice that this bound is lower than the $O^{*}\left(\sqrt{2}^{n}\right)$ on graphs of average degree 3 or less from Proposition 5. In our analysis, we use the following result, established by Reed in 1996:

Theorem 4 [14] On graphs of minimum degree 3, the size of MIN DOMINATING SET is not greater than $3 n / 8$.

We consider separately each connected component that contains at least a vertex of degree 3 . Notice the graph we consider may have vertices of degree 2 or less, so we complete the graph by adding edges between them until they all have degree at least 3 . No vertex that was already at the beginning of the process of degree 3 shall receive a new neighbor this way.

Then, we run an exact algorithm to find a MIN DOMINATING SET on the modified graph. This subset, namely $\mathcal{D}$, has a size at most $3 n / 8$. $\mathcal{D}$ is perhaps not a dominating set in $G$, but it is adjacent to all vertices of degree 3 . That means, once we have branched on any $v \in \mathcal{D}$, the remaining graph contains only vertices of degree 2 or less.

Of course there is an additive factor to our running time, that is complexity of solving MIN DOMINATING SET on a graph where any vertex but a finite number have degree 3. Using algorithm by [Fomin \& Hoie, 2006, [4]], we see that may be found in $O^{*}\left(2^{0.265 n}\right) \subset O^{*}\left(2^{3 n / 8}\right)$.

An rather immediate corollary is that any problem that matches vertex branching and is polynomial on graphs of maximum degree 2 can be solved on graphs of maximum degree $\Delta$ with running time $O^{*}\left(2^{n\left(1-(5 / 8)^{\Delta-2}\right)}\right)$. Unfortunately, for $\Delta \geq 4$, result from Proposition 5 overlap this one.

In case we can only make the weaker hypothesis that the problem is polynomial on totally disconnected graphs (in fact, we need a somewhat stronger hypothesis, namely, polynomiality on collection of bounded cliques), it is still possible to improve the $O^{*}\left(2^{3 n / 4}\right)$ result from Theorem 3 , if we know that our graph has maximal degree 3 instead of average degree 3 or less:

Proposition 7 Any problem that matches vertex branching and is polynomial on collections of cliques of bounded cardinality can be solved on graphs of maximum degree 3 within time $\mathrm{O}^{*}\left(2^{2 n / 3}\right)$.

If $G(V, E)$ contains a collection of 4-cliques, namely $K$, we first consider $G^{\prime}\left(V^{\prime}, E^{\prime}\right)=G[V \backslash K]$. We can find in polynomial time a 3-coloring of $G^{\prime}$. One of the three colors is an independent set $S$ of size at least $\left|V^{\prime}\right| / 3$. Any possible subset of $V^{\prime} \backslash S$ can be tested within time $O^{*}\left(2^{2\left|V^{\prime}\right| / 3}\right) \subset O^{*}\left(2^{2 n / 3}\right)$, and the remaining graph is a collection of cliques of size 1 or 4 .

Proposition 8 Any problem that matches vertex branching and is polynomial on graphs of maximum degree 2 can be solved on graphs of average degree $d \leq 3$ with running time $O^{*}\left(2^{21 n / 46}\right)$.

Proof. If $\Delta \leq 3$, the proposition is a consequence of Proposition 6. Otherwise we perform a sequence of branchings, at each time choosing a vertex of maximal degree, until our graph have maximum degree 3. Then, we consider the size of the remaining graph:

(i) If $n^{\prime}<20 n / 23$, we greedily branch on vertices of degree 3 until the graph has maximum degree 2.

(ii) Otherwise, we compute a MIN DOMINATING SET as described on Proposition 6 and branch on any vertex of it.

We form the finite sequence $\left\{n_{\Delta}, n_{\Delta-1}, \ldots, n_{4}\right\}$, where $n_{i}$ is the number of vertices of degree $i$ we branch on during first step of our algorithm. Fix $\sigma=\sum_{i \geq 4} n_{i}$. Since $i$ is the degree at the time we branch, not in the initial graph, the number of deleted edges is $\sum_{i \geq 4} i n_{i} \geq 4 \sigma$.

If hypothesis (i) holds, our algorithm is within time $O^{*}\left(2^{x}\right)$, where:

$$
\begin{aligned}
x & \leq \sigma+\frac{m-4 \sigma}{3} \\
& \leq \frac{n}{2}-\frac{1}{3} \times \frac{3 n}{23}=\frac{21 n}{46}
\end{aligned}
$$


On the other hand, if hypothesis (ii) is true, the time of the algorithm is within $O^{*}\left(2^{x^{\prime}}\right)$, and:

$$
\begin{aligned}
x^{\prime} & \leq \sigma+\frac{3(n-\sigma)}{8} \\
& \leq \frac{3 n}{8}+\frac{5}{8} \times \frac{3 n}{23}=\frac{21 n}{46}
\end{aligned}
$$

that completes the proof.

Thus, the following theorem holds:

Theorem 5 Any problem that matches vertex branching and is polynomial on graphs of maximum degree 2 can be solved on graphs of average degree bounded above by $d$ within time $O^{*}\left(2^{\frac{d-2723}{d+1} n}\right)$, for any $d \geq 3$.

Proof. The case $d=3$ is nothing but Proposition 8 . Assume that it is true for all values of average degree less than or equal to $d-1$. Then, thanks to Proposition 3, we can compute a solution when the average degree is less than or equal to $d$, within time $O^{*}\left(2^{\alpha_{d+1}}\right)$, where:

$$
\begin{aligned}
\alpha_{d+1} & =\frac{d \alpha_{d}+1}{d+1}=\frac{d \frac{d-1-27 / 23}{d}+1}{d+1} \\
& =\frac{d-27 / 23}{d+1}
\end{aligned}
$$

By induction, this is true for any $d$.

\subsection{Applying the bottom-up scheme for exact solution of the $f$-QIS problem}

Next proposition establishes the possibility to use a vertex branching method directly derived from the bottom-up scheme, for finding an optimal $f$-QIS within the time stated in Theorem 5.

Proposition 9 MAX QUASI-INDEPENDENT SET is polynomial on graphs of maximum degree 2 or less.

Proof. Let $\Delta$ the maximum degree of the graph we consider. If $\Delta=0$, we add vertices with minimum weight in a greedy manner, until condition $w_{0}+\sum_{v \in Q} w(v) \leq f\left(|Q|+q_{0}\right)$ gets violated, and find the optimal. If some vertex $v$ has degree 1 , let $u$ be its only neighbor.

- If $\delta(u)=1$, and, say, $w(u) \geq w(v)$, we can assume $u$ belongs to the solution only if $v$ does. Thus, we may remove edge $\{u, v\}$ and increase $w(u)$ by one. Of course this is symmetric between $v$ and $u$.

- If $\delta(u)=2$ and $w(u) \geq w(v)$, we can a fortiori remove the edge and increase $w(u)$ by 1 .

- Otherwise, $\delta(u)=2$ and $w(u) \leq w(v)-1$. Since $w(u)$ might increase by at most 1 (if the second neighbor of $u$ belongs to the sought optimal), we can safely remove $(u, v)$ and increase $w(v)$ by 1 .

One can also consider the case where no vertex has degree 1, meaning that $G$ is a set of cycles (at least one) and isolated vertices. Let $\left\{a_{i}\right\}_{i \leq k}$ be one of these cycle. If there exists some $i, w\left(a_{i}\right) \leq w\left(a_{i+1}\right)-1$, then we can remove $\left\{a_{i}, a_{i+1}\right\}$ and add 1 to $w\left(a_{i+1}\right)$. On the other hand, if for any $i, w\left(a_{i}\right)=w\left(a_{i+1}\right)$, then all vertices in the cycle are identical, some we can decide arbitrarily to remove $a_{1}, a_{2}$ and increase $w\left(a_{1}\right)$ by 1 . In any case, the cycle will become a path, and thus we can remove all its edges by successively disconnecting leaves, as explained above.

It follows that:

Corollary 4 Finding a maximum $f$-QIS on graphs of average degree $\leq d$ can be done within time $O^{*}\left(2^{\frac{d-27 / 23}{d+1} n}\right)$.

For instance, MAX QUASI-INDEPENDENT SET is graphs of average degree 3 can be solved in time $O^{*}\left(2 \frac{21}{46} n\right)$, while in graphs of average degree 4 , the corresponding time is $O^{*}\left(2^{\frac{13}{3}} n\right)$. 


\section{Approximation algorithms}

\subsection{Approximation of $\gamma-\mathrm{QIS}$}

\subsubsection{When $\gamma$ is bounded}

When $\gamma$ is bounded from below by a fixed constant, then things are rather optimistic, since the following result holds.

Theorem 6 Consider the $\gamma-Q I S$ problem when $\gamma$ is bounded below by a positive constant $c$. For any fixed $k \leq \Delta, a$ solution of size at least $k / \Delta$ the optimal can be computed within polynomial time.

Proof. Assume w.l.o.g. that $\Delta>2$ and the graph of the instance is not a collection of cliques (otherwise, the optimum can be found in polynomial time).

Under these assumptions, there is always a $\Delta$-coloring of the graph and it can be found in polynomial time. Let $S$ be a color class of the greatest size $s$; recall that $s \geq n / \Delta$. If $s \geq k / c$, then $S$ will be a $\gamma-$ QIS with the desired property, since by Lemma 2 we get

$$
\frac{q^{*}}{s} \leq \frac{\Delta / \gamma}{k / c} \leq \frac{\Delta / c}{k / c}=\frac{\Delta}{k}
$$

where $q^{*}$ is the size of the optimal $Q^{*}$.

Otherwise, $S$ has bounded finite size, so we can enumerate all subsets of size $k s$ or less within polynomial time. If $q^{*}<k s$, we come with the optimal; else, by Lemma 1 we know that there exists a $\gamma-Q I S Q \subset Q *$ of size $q=k s$. For that set, it is

$$
\frac{q^{*}}{q} \leq \frac{n}{k s} \leq \frac{n}{k \frac{n}{\Delta}}=\frac{\Delta}{k}
$$

and the result yields.

Corollary 5 If $\gamma$ is bounded below by some positive constant, then $\gamma-Q I S$ is polynomial for graphs with bounded degree.

\subsubsection{A greedy algorithm}

In this subsection, a greedy algorithm for computing a $\gamma$-QIS is discussed; the solution is initialized to some independent set $S$, and at each step a vertex of minimum degree to the current solution is being inserted; the insertions keep on, until the largest solution respecting the sparsity specification is reached.

procedure $\gamma_{-} \mathrm{QIS}(G(V, E)$ : graph, $0 \leq \gamma \leq 1$ : real, $S \subseteq V$ : some independent set)

$$
Q \leftarrow S
$$

$Q^{\prime} \leftarrow S$;

while $\left(\left|Q^{\prime}\right| \leq|V|\right)$

pick $v \in V \backslash Q^{\prime}$ such that $\delta_{Q^{\prime}}(v)=\min$ (break ties arbitrarily)

$Q^{\prime} \leftarrow Q^{\prime} \cup\{v\}$;

if $\left(\left|E\left[Q^{\prime}\right]\right| \leq \gamma\left(\begin{array}{c}\left|Q^{\prime}\right| \\ 2\end{array}\right)\right)$ and $\left(|Q| \leq\left|Q^{\prime}\right|\right)$

$$
Q \leftarrow Q^{\prime} \text {; }
$$

endif;

endwhile;

$\operatorname{return}(Q)$;

end $\gamma_{-}$QIS

Obviously, $\gamma$-QIS always returns a solution, if $S$ is set to some $\gamma-$ QIS (any independent set in $G$, for instance the empty set, would do).

As it has already been mentioned, the non-hereditary character of $\gamma-Q I S$ is reflected to the algorithm by the fact that it may be that some $Q^{\prime}$ produced during the execution of the algorithm is unfeasible while after some later vertex-insertions it may become feasible. This non-hereditary character of the problem is a major difficulty for a more refined analysis of algorithm $\gamma_{-}$QIS. The following lemma gives a lower bound on the size of the solutions returned this algorithm. 
Lemma 5 Let $q$ be the size of the $\gamma$-QIS returned by the algorithm, where S has been initalized to some independent vertex set. It is $q>\frac{\alpha-1}{\sqrt{1-\gamma}}$ with $\alpha$ the size of $Q$ during the last step of the algorithm's execution before the first edges insertion.

Proof. Let $E[Q]$ be the number of edges in the solution $Q$. By the definition of algorithm $\gamma_{-} \mathrm{QIS}$, if $S$ is initialized to an independent set, the first $\alpha$ vertices inserted into the solution form a maximal independent set. Then $q=\alpha+\kappa$, for some $\kappa, 0 \leq \kappa \leq n-\alpha$, ie after the insertion into the solution of the first $\alpha$ vertices which are independent of each other, $v_{1}, \ldots, v_{\kappa}$ have been inserted. Let $\delta_{Q_{i}}\left(v_{i}\right)$ be the degree of $v_{i}$ to the solution at the moment of its insertion, that is to $Q_{i}$. Then, $E[Q]=\sum_{i=1}^{\kappa} \delta_{Q_{i}}\left(v_{i}\right)$.

Notice that $\delta_{Q_{1}}\left(v_{1}\right) \leq \alpha$ and $\forall i, 2 \leq i \leq \kappa, \delta_{Q_{i}}\left(v_{i}\right) \leq \alpha+i-1$; hence, it is $E[Q] \leq \sum_{i=1}^{\kappa}[\alpha+(i-1)]=\kappa \alpha+\frac{\kappa(\kappa-1)}{2}$.

Assume w.l.o.g. that the solution computed is not a the whole graph (in which case it is trivially optimal). Then, by the definition of $\gamma_{-}$QIS, for any other candidate solution $Q^{\prime}$ containing $Q$ and having size $\alpha+\kappa+l$ for some $l \geq 1$, it is

$$
\begin{gathered}
\gamma \frac{(\alpha+\kappa+l)(\alpha+\kappa+l-1)}{2}<E\left[Q^{\prime}\right]=\sum_{i=1}^{\kappa+l} \delta_{Q_{i}}\left(v_{i}\right) \leq \sum_{i=1}^{\kappa+l}[\alpha+(i-1)]=(\kappa+l) \alpha+\frac{(\kappa+l)(\kappa+l-1)}{2} \Rightarrow \\
\gamma \frac{(\alpha-1+\kappa+l)(\alpha+\kappa+l)}{2}<(\kappa+l) \alpha+\frac{(\kappa+l)(\kappa+l-1)}{2}
\end{gathered}
$$

Setting $a=\alpha-1, k=\kappa+l$, inequality (4) is written

$$
\begin{array}{r}
\gamma(a+k)(a+k+1)<2 k(a+1)+k(k-1) \Leftrightarrow(1-\gamma) k^{2}+[2(1-\gamma) a+1-\gamma] k-\gamma a(a+1)>0 \Leftrightarrow \\
(1-\gamma) k^{2}+(1-\gamma)(2 a+1) k-\gamma a(a+1)>0
\end{array}
$$

Solving inequality (5) with respect to $k$, we get that

$$
\begin{array}{r}
k>-a-\frac{1}{2}+\frac{\sqrt{(1-\gamma)^{2}(2 a+1)^{2}+4(1-\gamma) \gamma a(a+1)}}{2(1-\gamma)} \\
k>-a-\frac{1}{2}+\frac{\sqrt{(1-\gamma)\left[(1-\gamma)\left(4 a^{2}+4 a+1\right)+4 \gamma a(a+1)\right]}}{2(1-\gamma)} \\
k>-a-\frac{1}{2}+\frac{\sqrt{(1-\gamma)\left(4 a^{2}+4 a+1-\gamma\right)}}{2(1-\gamma)}
\end{array} \Leftrightarrow
$$

Inequality (6) yields finally

$$
\begin{array}{r}
\kappa+l+\alpha>\alpha-(\alpha-1)+\frac{\alpha-1}{\sqrt{1-\gamma}} \Leftrightarrow \\
q=\kappa+\alpha>1-l+\frac{\alpha-1}{\sqrt{1-\gamma}},
\end{array}
$$

with the last inequality (7) holding also for $l=1$, hence

$$
q>\frac{\alpha-1}{\sqrt{1-\gamma}} \Rightarrow q \geq\left\lceil\frac{\alpha-1}{\sqrt{1-\gamma}}\right\rceil .
$$

that completes the proof.

Combining Lemma 5 and item 1 of Lemma 2, we finally get: 
Theorem 7 For the $\gamma$-QIS problem it is possible to find in polynomial time a solution of size $q$ such that

$$
\frac{q^{*}}{q} \leq \frac{\Delta \alpha_{\min }}{\alpha-1} \sqrt{1-\gamma}
$$

with $q^{*}$ the size of an optimal quasi-independent set and $\alpha_{\min }$ the size of a minimum independent dominating set of the input graph. This ratio tends to $\Delta \sqrt{1-\gamma}$.

Algorithm $\gamma$-QIS has been run on 20 randomly generated graphs of each size (10, 20 and 30 vertices); edges in an instance have been generated with a probability $p, 0.1<p<0.5)$. Optimal solutions have been computed with the exact method of Section 4 . Table 1 gives a summary of the experimental results obtained. It contains for every value of $\gamma$, the worst, best and average ratios and the percentage of optima returned by the algorithm.

\begin{tabular}{|c|c|c|c|c|}
\hline$\gamma$ value & Worst ratio & Best ratio & Average ratio & Optima as percentage of obtained solutions \\
\hline $0.2 d(G)$ & 0.667 & 1 & 0.947 & $66.667 \%$ \\
\hline $0.4 d(G)$ & 0.7 & 1 & 0.969 & $75 \%$ \\
\hline $0.6 d(G)$ & 0.75 & 1 & 0.983 & $83.333 \%$ \\
\hline $0.8 d(G)$ & 0.905 & 1 & 0.996 & $93.333 \%$ \\
\hline $1 / n$ & 0.667 & 1 & 0.96 & $75 \%$ \\
\hline $1 / \sqrt{n}$ & 0.778 & 1 & 0.98 & $85 \%$ \\
\hline $\log (n) / n$ & 0.778 & 1 & 0.973 & $76.667 \%$ \\
\hline
\end{tabular}

Table 1: Experimental performance of algorithm $\gamma$-QIS.

One may remark that the more the density of the sought subgraph comes close to the density of the instance, the best the quality of the returned solution is.

\subsubsection{Moderately exponential approximation for $\gamma$-QIS}

We finish the approximation section for $\gamma$-QIS by showing how it can be approximated within any constant ratio by exponential algorithms with running time better than that of an exact computation.

Theorem 8 For any $k \geq 1$, it is possible to compute a $\gamma$-QIS of size at least $1 / k$ of the optimal, within time $\mathrm{O}^{*}\left(2^{\left(\log _{2}(k+1)-k \log _{2} k /(k+1)\right) n}\right)$.

Proof. We enumerate every subset of size at most $n /(k+1)$ or at least $k n /(k+1)$, within time:

$$
\begin{aligned}
\sum_{i \leq n /(k+1)}\left[\left(\begin{array}{c}
n \\
i
\end{array}\right)+\left(\begin{array}{c}
n \\
n-i
\end{array}\right)\right] & \leq n\left(\begin{array}{c}
n \\
n /(k+1)
\end{array}\right) \\
& \leq n^{2} \frac{n^{n}}{(k n /(k+1))^{k n /(k+1)}(n /(k+1))^{n /(k+1)}} \\
& \leq n^{2}\left(\frac{k+1}{k^{k /(k+1)}}\right)^{n}
\end{aligned}
$$

We return the minimal one which is a $\gamma$-QIS. If we have for the size of the optimal $q^{*}$ that $q^{*} \geq k n /(k+1)$ or $q^{*} \leq n /(k+1)$, we come with the optimal. Otherwise, by Lemma 1 , we find a quasi-independent set $Q$ of size $q \geq n /(k+1)$, that means:

$$
\frac{q^{*}}{q} \leq \frac{k n /(k+1)}{n /(k+1)} \leq k
$$

and the proof is completed.

The following result exhibits a further link between $\gamma$-QIS and MAX INDEPENDENT SET.

Theorem 9 Given some algorithm that computes an exact solution for MAX INDEPENDENT SET on $G$ within time $O^{*}\left(c^{n}\right)$, for some constant $c$, a $\gamma$-QIS of size at least $1+\gamma n / 2$ can be computed within time $O^{*}\left(c^{n}\right)$. 
Proof. Suppose w.l.o.g. that $\gamma / 2<1$, (otherwise the optimal is trivial). Consider an optimal $\gamma-\mathrm{QIS} Q^{*}$ of size $q^{*}$. We note $\alpha^{*}(G)$ the size of a maximum independent set in G. Applying Turan's Theorem [2] to $G\left[Q^{*}\right]$, we get:

$$
\alpha^{*}\left(G\left[Q^{*}\right]\right) \geq \frac{\left(q^{*}\right)^{2}}{\left|E\left[Q^{*}\right]\right|+q^{*}} \geq \frac{q^{*}}{\gamma\left(q^{*}-1\right) / 2+1} \geq \frac{2}{\gamma+(2-\gamma) / q^{*}}
$$

Hence, by (10), using the optimal solution returned by the algorithm as a $\gamma-\mathrm{QIS}$, guarantees the following ratio:

$$
\frac{q^{*}}{\alpha^{*}(G)} \leq \frac{q^{*}}{\alpha^{*}\left(G\left[Q^{*}\right]\right)} \leq \frac{\gamma q^{*}+2-\gamma}{2} \leq \frac{\gamma n}{2}+1
$$

that completes the proof.

\subsection{Approximation of $k-\mathrm{QIS}$}

In this section we deal with polynomial approximation of $k$-QIS. We propose a greedy algorithm for that purpose, based upon the same idea as $\gamma$-QIS presented above; however, $k$-QIS being a hereditary problem, the algorithm stops as soon as it finds the first vertex whose insertion violates the condition on the number of edges allowed in the solution.

Procedure $k \mathrm{QIS}(G(V, E)$ : Graph, $k$ : integer $\geq 0, S$ : some independent set)

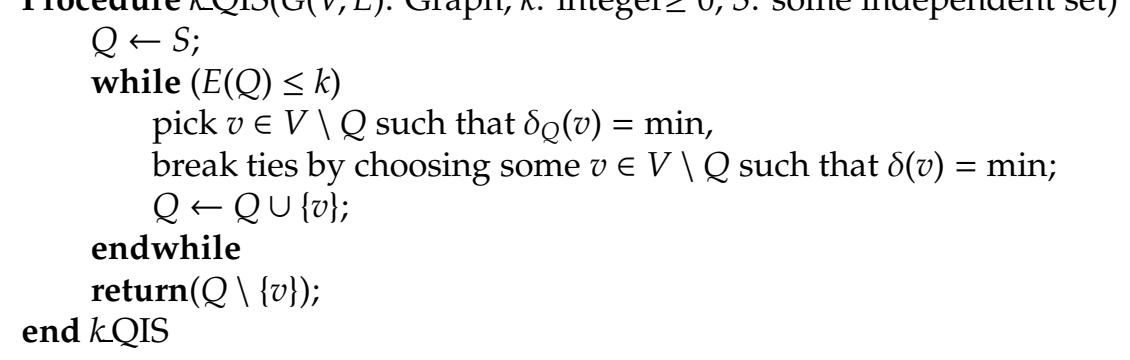

Let $Q$ be the solution computed by the algorithm; as in the proof of Lemma 5, we note by $q$ its size, and write $q=\alpha+x$, with $\alpha$ the size of $Q$ at the last step before the first edge insertions performed by the algorithm; we can suppose w.l.o.g. that this set is already computed before starting the algorithm. Let $\theta(S)=\max _{v \in V \backslash S}\left\{\delta_{S}(v)\right\}$. Clearly, for each of the remaining $x$ vertices, at most $\theta(S)$ edges are inserted into the QIS; thus, with $Q_{i}$ the solution after the $i$-th from the $x$ vertices insertions that brought some edges in, it is

$$
\begin{array}{r}
k=\sum_{i=1}^{x} \delta_{Q_{i}}\left(v_{i}\right) \leq \theta(S) x \Rightarrow k \leq \theta(S)(q-\alpha) \Leftrightarrow \\
q \geq \frac{k}{\theta(S)}+\alpha
\end{array}
$$

Let $Q^{*}$ be an optimal solution with $|Q|^{*}=q^{*}$ and consider the graph $G\left[Q^{*}\right]=\left(Q^{*}, E^{\prime}\right)$. Obviously, $\left|E^{\prime}\right| \leq k$. The set $Q^{*}$ can be seen as the union of two sets $S^{*}$ and $T$, where $S^{*}$ is a maximum independent set of $G\left[Q^{*}\right]$ and $T=Q^{*} \backslash S^{*}$. On the other hand, $E^{\prime}$ is the union of the set $E_{T}^{\prime}$ of edges within the set $T$ and of the set $E_{S^{*}, T}^{\prime}$ of edges between $S^{*}$ and $T$. Obviously, $\left|E_{S^{*}, T}^{\prime}\right| \leq k$. Moreover, since the graph $\left(Q^{*}, E_{S^{*}, T}^{\prime}\right)$ is bipartite and connected (the set $S^{*}$ is maximal for inclusion in both $G\left[Q^{*}\right]$ and $\left(Q^{*}, E_{S^{*}, T}^{\prime}\right)$ ), the fact that $\left|E_{S^{*}, T}^{\prime}\right| \leq k$ implies $|T| \leq k$. So, $Q^{*} \leq S^{*}+k \leq \alpha^{*}+k$, where $\alpha^{*}$ is the size of a maximum independent set in the graph $G$. Combining this bound with (11), we finally get the following.

Theorem 10 For the $k$-QIS problem it is possible to find in polynomial time a solution of size $q$ such that

$$
\frac{q^{*}}{q} \leq \frac{\alpha^{*} \theta(S)+k \theta(Q)}{\alpha \theta(Q)+k} \leq \max \left\{\frac{\alpha^{*}}{\alpha}, \theta(Q)\right\}
$$

where $q^{*}$ is the size of the optimal, $\alpha^{*}$ is the size of a maximum independent set in $G$ and $\alpha$ the size of some independent set.

In order to be more precise, let us first recall that the best approximation ratio (as function of $\Delta$ ) known for MAX INDEPENDENT SET is $(\Delta+2) / 3$ and is guaranteed by the natural greedy MAX INDEPENDENT SET-algorithm [7]. 
Suppose first that $\theta(Q) \geq 3$. Then, by item 2 of Lemma 2, the approximation ratio of algorithm $k$ QIS is bounded from above by $\Delta / 3$. Assume now that $\theta(Q) \leq 3$. Then, by Theorem 10 , the approximation ratio of the algorithm is bounded above by:

$$
\max \left\{\frac{\alpha^{*}}{\alpha}, 6\right\} \leq \frac{\alpha^{*}}{\alpha} \leq \frac{\Delta+2}{3}
$$

and the following holds.

Corollary $6 k$-QIS is approximable in polynomial time within ratio $\Delta+2 / 3$.

Another set of tests have been implemented in order to experimentally observe the behavior of performance of algorithm $k$ QIS presented for several values of $k$. As for the case of $\gamma$-QIS, we have used the exact method of Section 4 to compute optimal solutions of the test instances. Instances have been generated randomly, following a probability $p, 0.1<p<0.5$ for an edge to be present in the instance graph. The tests indicate that the algorithm performs fairly well in small instances. A summary of the obtained results is given in Table 2.

\begin{tabular}{|c|c|c|c|c|}
\hline$k$ value & Worst ratio & Best ratio & Average ratio & Optimal as percentage of obtained solutions \\
\hline $2 \sqrt{m}$ & 0.93 & 1 & 0.99 & $90 \%$ \\
\hline$\sqrt{n}$ & 0.83 & 1 & 0.98 & $80 \%$ \\
\hline $\log (m)$ & 0.83 & 1 & 0.98 & $80 \%$ \\
\hline $\log (n)$ & 0.83 & 1 & 0.97 & $75 \%$ \\
\hline$m / 2$ & 0.96 & 1 & 0.99 & $92.5 \%$ \\
\hline$m / 3$ & 0.96 & 1 & 0.99 & $95 \%$ \\
\hline$n / 2$ & 0.90 & 1 & 0.99 & $90 \%$ \\
\hline$n / 3$ & 0.90 & 1 & 0.99 & $87.5 \%$ \\
\hline
\end{tabular}

Table 2: Experimental performance of algorithm $k \mathrm{QIS}$

\section{Conclusions}

In this paper, we have presented the quasi-independent set problem that consists in searching in a graph for a maximum size subgraph that satisfies a suitably defined sparsity condition; according to the definition of the latter, this problem is formalized as the $f-$ QIS problem, or the $\gamma-$ QIS, or finally $k-$ QIS problem. An exact method for computing an optimal $f$-QIS-solution within time $O^{*}\left(2^{\frac{d-27 / 23}{d+1} n}\right)$ in graphs of average degree bounded above by $d$ has been designed, together with polynomial algorithms for finding approximate $\gamma$-QIS- and $k$-QIS-solutions, respectively. It has been shown that MAX QUASI-INDEPENDENT SET is polynomial in forests and split graphs, while it is NP-hard in bipartite graphs. Determining its complexity in other graph-classes, mainly those where MAX INDEPENDENT SET is polynomial, is an interesting matter of ongoing research. The experimental results obtained suggest that the greedy algorithms for $\gamma-$ QIS and $k-$ QIS perform better than it can be shown by their theoretical analysis. However, we believe that in order to refine them, one has to develop non-combinatorial arguments; such a task is another promising perspective for future work.

Finally, other ideas of generalizing the approach of the greedy algorithms for $\gamma-$ QIS and $k$-QIS could be tested. Indeed, these algorithms are based upon the fact that the (current) solution $Q$ can be merged with another (in our case, a selected single vertex $v$ ) to form a new, larger one (this is possible whenever $\left.\delta_{Q}(v) \leq \gamma \gamma q\right)$. As subtle point in algorithm $\gamma_{-}$QIS is that it allows for "temporary" violations of the sparsity measure $Q$, if they can be repaired after a number of greedy vertex insertions. However, this is rarely the case: although it is possible to find such instances, none has been generated in experimental tests. A more general algorithm could be based on merging of a current solution $Q$ with another, disjoint one $Q^{\prime}$ (the possibility condition being generalized to asking for $\left.\delta_{Q}\left(Q^{\prime}\right) \leq \gamma q q^{\prime}\right)$.

\section{References}

[1] J. Abello, M.G.C. Resende, and S. Sudarsky. Massive Quasi-Clique detection. In Proceedings of LATIN 2002, LNCS 2286, Springer-Verlag, 598-612. 
[2] C. Berge. Graphs and hypergraphs. North Holland, Amsterdam, 1973.

[3] V. Boginski, S. Butenko and P. Pardalos. Mining market data: a network approach. Computers and Operations Research (2005). In press, available online at http://www . sciencedirect. com/.

[4] F.V. Fomin and K. Hoie. Pathwidth of cubic graphs and exact algorithms, Information Processing Letters, 97(5): 191-196, 2006.

[5] A.V. Goldberg. Finding a Maximum Density Subgraph. Technical report UCB CSD 84/171, University of California, Berkeley CA, 1984.

[6] M.M. Halldórsson. Approximating the minimum maximal independence number. Information Processing Letters, 46(4): 169-172, 1993.

[7] M.M. Halldórsson and J. Radhakrishnan. Greed is good: approximating independent sets in sparse and bounded-degree graphs. In Proceedings of STOC 1994, 439-448.

[8] L.H. Hartwell, J.J. Hopfield, S. Leibler, and A.W. Murray. A.W. From molecular to modular cell biology. Nature, 402: C47-C52, 1999.

[9] D.S. Hochbaum and O. Goldschmidt. k-edge subgraph problems. Discrete Applied Mathematics, 74(2): 159-169, 1997.

[10] A. Jagota, G. Narasimhan and Ľ. Šoltés. A generalisation of Maximal Independent Sets. Discrete Applied Mathematics, 109(3): 223-235, 2001.

[11] M.S. Krishnamoorthy and N. Deo. Node-deletion NP-complete problems, SIAM J. Comput., 8: 619-625, 1979.

[12] C. Lund and M. Yannakakis. The approximation of maximum subgraph problems. In Proceedings of ICALP 1993, LNCS 700, Springer-Verlag, 40-51.

[13] J.C. Picard and M. Queyranne. Selected applications of minimum cuts in networks. Information Systems and Oper. Res., 20: 394-422, 1982.

[14] B. Reed. Paths, stars and the number three. Combinatorial Probabilistic Computing, 5: 277-295, 1996.

[15] M. Yannakakis and J. Lewis. The Node-Deletion Problem for Hereditary Properties is NP-Complete. Journal of Computer and System Sciences 20(2): 219-230, 1980.

[16] V. Zissimopoulos. Private communication.

[17] D. Zuckerman. Linear degree extractors and the inapproximability of max clique and chromatic number. In Proceedings of STOC 2006, 681-690. 